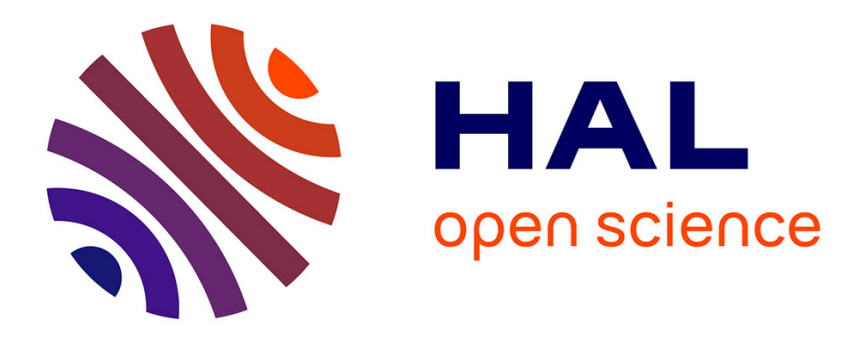

\title{
Study of Ice Crystal Orientation in Cirrus Clouds based on Satellite Polarized Radiance Measurements
}

Vincent Noel, Hélène Chepfer

\section{To cite this version:}

Vincent Noel, Hélène Chepfer. Study of Ice Crystal Orientation in Cirrus Clouds based on Satellite Polarized Radiance Measurements. Journal of the Atmospheric Sciences, 2004, 61, pp.2073-2081. hal-00147432

\section{HAL Id: hal-00147432 \\ https://hal.science/hal-00147432}

Submitted on 17 May 2007

HAL is a multi-disciplinary open access archive for the deposit and dissemination of scientific research documents, whether they are published or not. The documents may come from teaching and research institutions in France or abroad, or from public or private research centers.
L'archive ouverte pluridisciplinaire HAL, est destinée au dépôt et à la diffusion de documents scientifiques de niveau recherche, publiés ou non, émanant des établissements d'enseignement et de recherche français ou étrangers, des laboratoires publics ou privés. 


\title{
Study of ice crystal orientation in cirrus clouds based on satellite polarized radiance measurements
}

\author{
Vincent Noel ${ }^{1}$, Hélène Chepfer ${ }^{2}$ \\ ${ }^{1}$ Analytical Services and Materials, Hampton, VA USA \\ ${ }^{2}$ Laboratoire de Meteorologie Dynamique, Ecole Polytechnique, \\ Palaiseau France
}

February 3, 2004

Submitted to the Journal of Atmospheric Sciences

corresponding author :

Vincent Noel <v.r.noel@larc.nasa.gov>

Mail Stop 435

NASA Langley Research Center

Hampton, VA 23681-2199, USA 


\begin{abstract}
The goal of this paper is to retrieve information about ice particle orientation in cirrus clouds. This is achieved by comparing simulations of sunlight reflection on a cirrus cloud with measurements of polarized radiances from the spaceborne instrument POLDER-1/ADEOS-1. Results show that horizontal orientation of crystals can be spotted by the presence of a local maximum of polarized radiance in the direction of specular reflection. The angular width of the local maximum is shown to contain information on the particle maximum deviation angle, while the maximum intensity can provide information on particle shape and relative concentrations of ice crystals, horizontally and randomly-oriented. The study of 31 ice cloud cases show that in $80 \%$ of them, the deviation angle is less than $3^{\circ}$. Also, the relative concentration of horizontally-oriented crystals is less than $21 \%$, depending on the angular distribution used for crystal deviation.
\end{abstract}




\section{Introduction}

Cirrus clouds cover permanently more than $30 \%$ of the Earth's surface (Wylie et al, 1994), and as such have a strong influence on the planet's radiative balance (Stephens et al, 1990). As they are made of ice crystals, their influence strongly depends on their microphysical properties. However, for cirrus clouds such properties show a very high variability, and due to the high altitude of these clouds in situ data is only scarcely available. As a consequence, the overall cirrus radiative impact is still uncertain, with one of the largest sources of uncertainty remaining the microphysical properties, and especially the particles orientation in space.

Usually, studies of crystal properties inside cirrus clouds implicitely assume a random orientation for the particles. This type of model has symmetry properties that allow great simplification in computations of crystals optical properties and radiative transfer. Nowadays, this model is still used in the vast majority of radiative transfer simulations used to parametrize cirrus clouds models. However, it cannot give a satisfactory explanation for all optical displays created by these clouds, and could lead to possible bias when estimating their radiative impact.

First observations of preferential orientation of ice crystals were done through stro- 
boscopic measurements (Magono, 1953), refined several years later by studying droplets accretion on falling ice particles (Ono, 1969). These first studies revealed that specific shapes of ice crystals, such as plates, columns and dendrites, oriented themselves while falling, in order to offer maximum resistance to the air. As in-situ observation of this dynamic behavior is not possible, remote sensing was gradually introduced in the studies that followed. Radar (Hendry and McCormick, 1976) and direct photography (Sassen, 1980) were used first, however the instrument that generated the most studies of the orientation phenomenon is the lidar (Platt et al, 1978; Sassen, 1980; Thomas et al, 1990; Sassen and Takano, 2000; Noel et al, 2002), thanks to its ability to detect optically thin clouds and its sensitivity to light polarization.

Theoretical and laboratory studies (Jayaweera and Mason, 1965; Podzimek, 1968;

List and Schemenauer, 1971; Lynch et al, 1994; Klett, 1995) gave further insight into this behavior. Computer simulations (Takano and Liou, 1989a) showed that oriented crystals could increase the cloud albedo by as much as $30 \%$, leading to important modifications on the cloud radiative effect (Takano and Liou, 1989b). Recent observations (Sassen et al, 2003) suggest the crystals orientation could even have a potential feedback effect on particle generation inside the cloud. These results confirm the possible impact of this parameter on climate prediction. Unfortunately, the frequency and con- 
centration of horizontally-oriented crystals at a global scale in ice clouds is still largely unknown.

In order to estimate the overall impact of crystals orientation on cloud radiative effect, studies have to be pursued on a global scale, which is only possible through satellite monitoring. Following this approach, radiances observations from satellite instruments have been found to convey information about cirrus clouds microphysical properties (Chepfer et al, 2000, 2002; Liou and Takano, 2002; Masuda et al, 2002). More specifically, POLDER-1 (POLarization and Directionality of the Earth's Reflectance) observations of sunlight scattering upon ice clouds were recently used to estimate the frequency of the preferred orientation of crystals in cirrus (Chepfer et al, 1999). The present paper focuses on the cases where oriented crystals were actually observed. Measurements of normalized polarized radiances are compared to results of ray-tracing simulations to retrieve informations about the crystals orientation behavior.

The observations and the simulations are presented in Sect. 2, followed by the sensivity study of simulated normalized polarized radiances to crystal microphysical properties (Sect. 3). Based on these findings, the horizontally-oriented crystals maximum deviation angle and relative concentrations are retrieved in Sect. 4. Discussion and conclusion are given in Sect. 5. 


\section{Observed and simulated radiances}

\subsection{Observed radiances from satellite}

The preferential orientation of ice crystals in a cirrus cloud creates physical anisotropy that can be detected with multi-directional observations. For example, in presence of horizontally-oriented plates, bidirectional polarized radiances show a distinctive highintensity peak in the direction of specular reflection. This distinctive behavior has been used to estimate the frequency of horizontal orientation of crystals in cirrus clouds (Chepfer et al, 1999), using observations of normalized polarized radiances reflected by the atmosphere from the POLDER-1 spaceborne radiometer.

POLDER-1 (Deschamps et al, 1994) flew on the ADEOS-1 satellite from August 96 to June 97 and, up to now, seems the best suited instrument to retrieve information about crystal orientation in cirrus cloud. This radiometer measured normalized radiances $L_{n}\left(\theta_{v}, \varphi_{v}\right)$ and normalized polarized radiances $L_{n, p}\left(\theta_{v}, \varphi_{v}\right)$ for a given ground or cloud pixel from up to 14 viewing directions, defined by their zenith and azimuthal angles $\theta_{v}$ and $\varphi_{v}$ (Hagolle et al, 1999) :

$$
L_{n}\left(\theta_{v}, \varphi_{v}\right)=\frac{\pi L\left(\theta_{v}, \varphi_{v}\right)}{\cos \theta_{s} E_{s}} \quad L_{n, p}\left(\theta_{v}, \varphi_{v}\right)=\frac{\pi L_{p}\left(\theta_{v}, \varphi_{v}\right)}{\cos \theta_{s} E_{s}}
$$


with $\theta_{s}$ the solar zenithal angle and $E_{s}$ the solar irradiance at the top of the atmosphere.

$L_{n}\left(\theta_{v}, \varphi_{v}\right)$ and $L_{n, p}\left(\theta_{v}, \varphi_{v}\right)$ were measured at several visible wavelengths (443nm-865nm).

In order to minimize bias due to molecular scattering, this study uses the $865 \mathrm{~nm}$ channel.

To simulate $L_{n}\left(\theta_{v}, \varphi_{v}\right)$ and $L_{n, p}\left(\theta_{v}, \varphi_{v}\right)$, multiple scattering effects have to be considered (Sect. 2.2).

\subsection{Normalized polarized radiance and multiple scattering}

Multiple scattering has been shown to have a significant impact on light scattered by randomly-oriented particles (Trankle and Greenler, 1987). However, in presence of horizontally-oriented particles, its impact on normalized radiances is still not correctly quantified, even if its influence on plane albedo has been estimated (Takano and Liou, 1989b). In such a case, a reasonable guess would be to assume this impact is similar in preferential and random orientation. For this last case, studies of multiple scattering effects on total and polarized radiances (Goloub et al, 1994) have shown that, while the total radiance is strongly affected by multiple scattering, polarized radiance is mainly formed by the first scattering event, with saturation quickly taking place after a few events and no further influence from following events. Hence, if an observed cloud 
has a optical depth high enough for the polarized radiance to reach saturation, the reflected normalized polarized radiance does not depend on the cloud optical depth anymore.

In such cases, it is possible to write a single-scattering approximation of normalized polarized radiance from the general radiative transfer equation (Goloub et al, 1994) :

$$
L_{n, p}\left(\theta_{v}, \varphi_{v}\right)=\frac{\cos \theta_{s}}{4\left(\cos \theta_{s}+\cos \theta_{v}\right)} \sqrt{Q^{2}+U^{2}+V^{2}}
$$

with $Q, U$ and $V$ are the second, third and fourth elements of the scattered Stokes vector (Van de Hulst, 1957). This approximation has already been widely used in the litterature (C-Labonnote et al, 2000, 2001; Goloub et al, 2001; Riedi et al, 2000, 2001). In order to ensure its validity, all the cases selected for the present paper (Sect. 4) show a high optical depth.

\subsection{Simulated radiances from modeled crystals}

Following Eq. 2, the scattered Stokes vectors are sufficient to simulate normalized polarized radiances $L_{n, p}\left(\theta_{v}, \varphi_{v}\right)$ for any viewing direction in space. The Stokes vectors were simulated using a ray-tracing code (Noel et al, 2001), for clouds composed of preferentially oriented crystals. 
As a first approximation, the modelled ice crystals were shaped as hexagonal-based plates with variable aspect ratios $A=\frac{l}{2 r}$ (with $l$ the crystal length and $r$ the radius of the hexagonal base). This simple shape seems to accurately reproduce the scattering behavior of cirrus clouds (Heymsfield and Platt, 1984). The plates are also the particles more likely to exhibit an preferential orientation with a specific optical signature (Ono, 1969; Sassen and Takano, 2000). Preferential orientation is taken into account in the model through the deviation angle $\alpha$ from the horizontal plane. As the general distribution of $\alpha$ is still unknown, a gaussian and a square probability functions are considered, both centered on $\alpha=0$, with a standard deviation $\alpha_{\max }$. In this framework, $\alpha_{\max }$ gives an indication of the average deviation of ice crystals from the horizontal plane, with $\alpha_{\max }=0^{\circ}$ meaning a perfect horizontal orientation.

Once the crystals parameters $A$ and $\alpha_{\max }$ are chosen, and the solar zenithal angle $\theta_{s}$ is fixed, the ray-tracing simulation delivers the entire $4 \times 4$ scattering matrix $M\left(\theta_{v}, \varphi_{v}\right)$ of the modelled crystal for any viewing direction in space. As incident sunlight is unpolarized (i.e. its Stokes vector is $\mathbf{I}=(1,0,0,0))$, the Stokes vector of the scattered light (Van de Hulst, 1957) is easily obtained by : 


$$
\mathbf{I}_{\mathbf{s}}\left(\theta_{v}, \varphi_{v}\right)=M\left(\theta_{v}, \varphi_{v}\right) \cdot(1,0,0,0)=\sum_{i=1}^{4} M_{i, 1}\left(\theta_{v}, \varphi_{v}\right)
$$

Normalized polarized radiances are then easily calculated using Eq. 2.

\section{Study of simulated polarized radiances}

\subsection{Sensitivity study}

For the sake of simplicity, in this sensitivity study scattering is considered in the primary scattering plane $\left(\varphi_{v}=\varphi_{s}=0\right)$, with a solar incidence angle arbitrarily fixed at $\theta_{s}=43^{\circ}$. For actual case studies (Sect. 4) the exact scattering plane and incidence angle measured by POLDER will be used.

As an example, the simulated normalized polarized radiance $L_{n, p}$ reflected by the cloud is plotted in Fig. 1 as a function of the zenith angle $\theta_{v}$, for three square distributions of deviation angles with $\alpha_{\max }=1,3,5^{\circ}$, and two crystal aspect ratios $A=0.05$ and 0.5 . The comparatively low $L_{n, p}$ for randomly-oriented crystals is plotted in bold line. In these curves, $L_{n, p}$ shows a local maximum in the direction of specular reflection $\left(\theta_{v}=43^{\circ}\right)$, which is not present for randomly-oriented particles.

Similar computations using a gaussian distribution for the deviation angle $\alpha$ give 
a similar shape for $L_{n, p}$ and have not been plotted in Fig. 1 for readability. However, properties of the simulated curves depend strongly on the chosen distribution (square or gaussian) and on the value of $\alpha_{\max }$. Similarly, the particle aspect ratio has a strong influence.

\subsubsection{Sensitivity to crystal maximum deviation angle}

The general shape of the curve is strongly dependent on the crystal maximum deviation angle $\alpha_{\max }$ (Fig. 1). For crystals with small deviations, the curve shows a very narrow peak of high intensity in the direction of specular reflection. If the crystals deviation increase, the maximum intensity quickly decreases, while its angular width increases. This behavior reaches its limit for randomly-oriented crystals : the local maximum totally disappears.

The peak angular width, at half-maximum intensity, is plotted in Fig. 2 as a function of the crystal maximum deviation angle $\alpha_{\max }$ for three different aspect ratios : 0.05 , 0.2 and 0.5 . For square distributions of the deviation angle $\alpha$, very small deviations $\left(\alpha_{\max } \simeq 1^{\circ}\right)$ show a local maximum with a $2^{\circ}$ angular width. This width increases very quickly with crystal deviation, gaining approximately $5^{\circ}$ for a $2^{\circ}$ increase in $\alpha_{\max }$. For gaussian distributions the angular widths are smaller (i.e. the peaks are narrower) by 
$1.5^{\circ}$ in average, due to the higher probability given to smaller angular deviations. If the deviations are too small (i.e. $\alpha_{\max }<0.5^{\circ}$ ), the angular width becomes too narrow to be measured. With both distributions, the curve is monotonic for small deviations $\left(\alpha_{\max }<6^{\circ}\right.$, angular width up to $\left.\simeq 15^{\circ}\right)$. The maximum deviation angle $\alpha_{\max }$ can then be linked to the angular width of the local maximum, independently from the aspect ratio.

The curve maximum intensity is plotted in Fig. 3 as a function of crystal aspect ratio $A$ for three maximum deviation angle $\alpha_{\max }: 1,2$ and $3^{\circ}$. The maximum intensity is very sensitive to the crystal deviations : small deviations lead to high intensities, and a $1^{\circ}$-increase in $\alpha_{\max }$ leads to a $50 \%$ fall in maximum intensity. In addition, the effect of crystal aspect ratio $A$ is more apparent on the maximum intensity (Fig. 3) than on the peak angular width (Fig. 2).

\subsubsection{Sensitivity to crystal shape}

The crystal aspect ratio $A$ has a more subtle impact on the polarized radiance. For small crystal deviations $\left(\alpha_{\max }\right.$ up to $\left.6^{\circ}\right)$, the aspect ratio has almost no influence on the curve angular width (Fig. 2). When deviations get larger $\left(\alpha_{\max }>6^{\circ}\right)$, the crystal aspect ratio begins to influence the curve angular width. This is equally valid without regard 
to the distribution chosen for $\alpha$.

The influence of crystal shape is more obvious when looking at the curve maximum intensity. For square distributions, low aspect ratios $(A<0.15)$ lead to high normalized

polarized radiances $L_{n, p}>8$ (Fig. 3). When the aspect ratio increases, the polarized radiances decreases, with a threshold for $0.2<A<0.3$ due to consecutive scattering events inside the crystal. There can be a factor 10 between local maximum intensities for very low $(A<0.05)$ and very high $(A \simeq 1)$ aspect ratios. For a gaussian distribution, the same behavior is observed; however, the maximum intensities are significantly increased, up to $400 \%$ for $\alpha_{\max }=1^{\circ}$. This will have important consequences on the retrieved microphysical properties (Sect. 3.2).

\subsubsection{Sensitivity to Crystal concentrations}

Until this point of the study, clouds were assumed to be only composed of horizontallyoriented crystals. However, it is possible that only a fraction of crystals are closely aligned with the horizontal plane, while the remaining crystals are randomly oriented. If a cloud is defined as a mix of horizontally-oriented crystals (noted by the subscript $h$ ) and randomly-oriented crystals (noted by the subscript $r$ ), the normalized polarized 
radiance $L_{n, p}$ scattered by the entire cloud will be given by (Sassen and Benson, 2001) :

$$
L_{n, p}=\frac{n_{h} L_{n, p, h}+n_{r} L_{n, p, r}}{n_{h}+n_{r}}
$$

where $n$ is the relative concentration of each particle bearing the relevant orientation, and $L_{n, p}$ the related radiance (Eq. 2). For randomly-oriented crystals there is no maximum of normalized polarized radiance in the direction of specular reflection, thus $L_{n, p, r} \ll L_{n, p, h}$ (typically, for a solar zenithal angle $\theta_{s}=43^{\circ}, L_{n, p, r}<0.014$ in the direction of specular reflection). If $L_{n, p, r}$ is neglected, the total normalized polarized radiance is given by $L_{n, p}=\frac{n_{h}}{n_{h}+n_{r}} L_{n, p, h}$. The local maximum of normalized polarized radiance will linearly decrease as the quantity of randomly-oriented particles increase.

\subsection{Retrieval of cloud microphysical properties}

Following the sensivity study (Sect. 3.1), microphysical properties of the studied cloud can be retrieved using observed normalized polarized radiances.

In a first step, the crystal maximum deviation angle $\alpha_{\max }$ can be retrieved from the angular width of the polarized radiance local maximum (Sect. 3.1.1), provided the deviations are less than $6^{\circ}$ wide. As gaussian distributions lead to slightly thinner peaks for a given $\alpha_{\max }$ (Fig. 2), stronger deviations will be required to produce a given 
angular width.

Once $\alpha_{\max }$ is known, the intensity of the polarized radiance local maximum can be estimated as a function of crystal aspect ratio $A$ and relative concentration of horizontallyoriented particles $n_{h}$ (Sect. 3.1.2 and 3.1.3). Gaussian distribution leads to higher intensities (Fig. 3), so lower concentrations will be required to produce a given intensity. These two values are correlated, and sets of possible values can be retrieved based on the actual polarized radiances measurements.

As an example, the maximum intensity of $L_{n, p}$ is shown in Fig. 4 as a function of crystal aspect ratio $A$ and horizontally-oriented crystals relative concentration $n_{h}$, for a square distribution of deviation angles with $\alpha_{\max }=1^{\circ}$. Using this figure, an observed polarized radiance maximum intensity can be linked to a range of correlated values for $A$ and $n_{h}$. In this specific case, cloud areas showing polarized radiances values greater than 3 should contain more than $30 \%$ of horizontally-oriented crystals, the exact value depending on their shape. 


\section{Studied case}

\subsection{Case study}

Each case must be studied for the actual viewing angles $\theta_{v}, \varphi_{v}$, for which the azimuth angle $\varphi_{v}-\varphi_{s}$ is rarely constant. Therefore, polarized radiances are considered as a function of the angle $\tau$ between the direction of specular reflection and the actual scattering direction. The local maximum of polarized radiance is therefore centered on $\tau=0^{\circ}$.

The case studied in this section was measured by the POLDER-1 radiometer at coordinates $60.25 \mathrm{~N}, 25.15 \mathrm{~W}$, through orbit 7352 over sea. In this case, sunlight reflected on an ice cloud with incident angle of $\theta_{s}=42^{\circ}$. Measurements of normalized polarized radiance are shown in Fig. 5 as a function of $\tau$. They show a local maximum of $L_{n, p} \simeq$ 0.5 in the direction of specular reflection (for $\tau \simeq 0^{\circ}$ ), while staying relatively low for other scattering directions. This behavior suggests the presence of horizontallyoriented crystals in the studied cloud.

Measurements of polarized radiances are very similar to results from ray-tracing simulations (Fig. 1). More specifically, the angular width and intensity of the local maximum lie within the same range. It should then be possible to retrieve the maxi- 
mum deviation angle and relative concentration of the horizontally-oriented crystals present in the cloud, following the steps shown in Sect. 3.2.

\subsection{Retrieval of the crystal maximum deviation angle}

The angular width of the peak at half maximum intensity is approximately $1.5^{\circ}$. When considering normalized polarized radiance, the angular width of a local maximum is linked to the maximum deviation angle of horizontally-oriented crystals in the cloud, with little regard to the quantity or shape of these crystals (Sect. 3.1.1). To retrieve the maximum deviation angle, simulations are first conducted with an arbitrary large crystal deviation (typically $\alpha_{\max }=60^{\circ}$ ). The simulated normalized polarized radiances are then compared to the actual observations, with respect to the exact scattering direction given by the angles $\theta_{v}, \varphi_{v}$ (Sect. 2.3). Based on the width difference between the simulated polarized radiances and the measured ones, an adjusted value of the crystal maximum deviation angle is used in a new round of simulations. This whole process is repeated until convergence is reached. The crystal maximum deviation angle is the one giving the best agreement between the simulated and measured radiances.

For the actual case, the retrieved crystal maximum deviation is $0.6^{\circ}$ considering a square angular distribution and $2.1^{\circ}$ considering a gaussian distribution. 


\subsection{Retrieval of horizontally-oriented crystals shape and relative con- centration}

When considering a local maximum of normalized polarized radiance created by horizontallyoriented crystals, its intensity is linked to the shape and relative concentration of these crystals with respect to the angular distribution of the crystal deviation (Sect. 3.1.3). As the crystals maximum deviation is obtained independently (Sect. 4.2), the shape and relative concentration of these crystals can be retrieved.

The retrieved crystal maximum deviations for the actual case are $0.6^{\circ}$ and $2.1^{\circ}$ (Sect. 4.2). Using the retrieved $\alpha_{\max }$ and the exact POLDER observation angle, local maximums of polarized radiances are simulated for different aspect ratios. For each aspect ratio, the relative concentration of horizontally-oriented particles $n^{h}$ is estimated (Sect.

3.2). These concentrations are shown in table 1 for both square and gaussian angular distributions.

Even if the relative concentration $n^{h}$ shows high variations for different crystal shapes (the retrieved concentration is between 5 and 6 times higher for $A=0.5$ than for $A=0.05$, depending on the deviation distribution), the concentration values stay overall very low, below $4 \%$. As was expected (Sect. 3.2), the use of gaussian distribution leads to lower concentrations. 


\subsection{Multiple cases}

The same analysis is conducted on 30 more cases where POLDER measurements show a local maximum of normalized polarized radiance, and there are enough measurements to determine the angular width. For each case, normalized polarized radiances are simulated for the exact observation angles $\theta_{v}, \varphi_{v}$, until the simulated peak width is consistent with the measured values.

The frequencies of retrieved maximum deviation angles are shown in Fig. 6 for square and gaussian distributions of deviation angles, with respective average values $1^{\circ}$ and $1.8^{\circ}$. In more than $80 \%$ of the cases, the retrieved maximum deviation angle is less than $3^{\circ}$. It follows that, in most cases, the ice crystals are almost perfectly horizontally-oriented, with slightly larger deviations more frequent for gaussian distributions ( $\simeq 40 \%$ of cases show $1^{\circ}<\alpha_{\max }<3^{\circ}$, against $\simeq 15 \%$ for square distributions).

Following the previous case study (Sect 4.3), once the maximum deviation angle is retrieved for a given case, polarized radiances are simulated for several aspect ratios $A$, estimating for each one the relative concentrations of horizontally-oriented ice crystals $n_{h}$. These values are shown for square (Fig. 7.a) and gaussian (Fig. 7.b) distributions of deviation angles, with average relative concentrations given in Table 2. The frequency 
of horizontally-oriented crystals is between 6.2 and $21.4 \%$ if a square distribution is assumed, and between 5.4 and $8.6 \%$ if a gaussian distribution is assumed. Moreover, for any aspect ratios the relative concentration of horizontally-oriented crystals has more than $60 \%$ probability to be under $10 \%$. The second highest frequency (Fig. 7.a and $\mathrm{b}$ ) is for a relative concentration between 10 and $20 \%$, but it is much lower (below $20 \%$ ). The chosen angular distribution has only a small influence here.

\section{Discussion and conclusion}

The present study shows that, in presence of horizontally-oriented crystals within a cirrus cloud, their maximum deviation angle can be retrieved from spaceborne measurements of polarized radiances. This angle is generally very low, below $3^{\circ}$ in most cases, with little influence from the deviation angle distribution. Previous studies used other ground-based observations to retrieve this parameter : Sassen (1980), through a statistical analysis of direct photography, found oriented ice crystals in cirrus clouds were more likely to bear a gaussian angular distribution, with a half-width angle below $1.4^{\circ}$ for most particle shapes and an maximum value of $2.5^{\circ}$. Later, most studies took advantage of the rapid variation of the lidar signal near the zenith direction in presence of oriented crystals : looking at the backscattered intensity, Platt et al (1978) 
found a maximum deviation near $0.5^{\circ}$, while Thomas et al (1990), by studying the more sensitive depolarization ratio, found a deviation angle no higher than $0.3^{\circ}$. However, these studies were mostly ground-based, and their conclusions are local. The present study draws consistent results from 31 cloud cases observed from satellite. Moreover, the concentration of oriented particles relative to the entire population of crystals, a parameter still unknown at this point, was found to be under $10 \%$ in most of the studied clouds. The application of this procedure to a high number of spaceborne measurements (from e.g. POLDER-2 or PARASOL) could give helpful insight into the orientation behavior of ice crystals in cirrus clouds, information that is otherwise very hard to get and still largely unknown.

However, there are still some limitations with this technique. For example, future progress in radiative transfer computations may show that the influence of multiple scattering effects can not be considered similar in isotropic and anisotropic media, as was assumed in the present study (Sect. 2.2). Moreover, oriented ice crystals were taken as plates, which is the most likely hypothesis. However, oriented hollow columns can produce specific optical signatures similarly to plate crystals (Sassen and Takano, 2000), and in the future the way to discriminate these two shapes has to be investigated. Also, POLDER-1 measurements themselves have inherent limitations, the 
more important one being their spatial resolution of $6 \times 6 \mathrm{~km}$. Even if still being able to witness a signature of crystals orientation at such a large scale confirms the potential radiative implications of this phenomenon, there could be large variations in $\alpha_{\max }$ over an area so large. By analyzing smaller cloud areas through other techniques, the spatial variability of crystal orientations could be established at smaller scales, assessing the representativity of POLDER-1 retrievals. Another problem occurs when the polarized radiances saturate the POLDER-1 sensors for some contiguous pixels. In such cases the retrieval is still possible, but the peak angular width is measured with a lower accuracy, which has an impact on the retrieved maximum deviation angle. Finally, the procedure shown in this paper only provides a range of correlated values : the relative concentration and aspect ratio (Sect 4.3). Although the variation of concentration with the supposed aspect ratio is quite small (less than $5 \%$ ), any external retrieval of crystal shapes would significantly increase the reliability and credibility of the concentration results. When considering future observations from the PARASOL instrument, the collocated CALLIOPE lidar from the CALIPSO mission (Winker and Wielicki, 1999) could provide useful information about particle shape, thus constraining the retrieval.

Even with these limitations, valuable information can be gained about crystals orientation in cirrus clouds from spaceborne polarized radiometric measurements. Up 
to now, it remains the only known technique for retrieving this property on a global scale. As a next step to this study, the retrieval technique presented in this article could be applied to data from two new spaceborne instruments. First, the POLDER-2 instrument, located on ADEOS-2, which is already in orbit, has begun as of January 2003 to take measurements. Second, the PARASOL mission, scheduled for launch in 2004, will provide another source of spaceborne POLDER measurements. Its location in the the constellation a satellites named Aqua-Train will allow interaction with other spaceborne instruments, with potential improvements to the retrieval technique discussed in the present article. These results will greatly improve our understanding of the physical behavior of cirrus particles over the planet.

Acknowledgements. The results presented in this paper were obtained using data from CNES's POLDER instrument onboard NASDA's ADEOS platform. The authors are grateful to F.-M. Breon for processing the POLDER-1/Level-0 data. 


\section{References}

C-Labonnote, L., G. Brogniez, J. C. Buriez, M. Doutriaux-Boucher, J. F. Gayet and A. Macke, Polarized light scattering by inhomogeneous hexagonal monocrystals: Validation with adeos-polder measurements. J. of Geophys. Res., 106(11): 1213912153, 2001.

C-Labonnote, L., G. Brogniez, M. Doutriaux-Boucher and J.-C. Buriez, Modeling of light scattering in cirrus clouds with inhomogeneous hexagonal monocrystals. comparison with in situ and adeos-polder measurements. Geophys. Res. Let., 27(1): 113$116,2000$.

Chepfer, H., G. Brogniez, P. Goloub, F. M. Breon and P. H. Flamant, Observations of horizontally oriented ice crystals in cirrus clouds with polder-1/adeos-1. J. of Quant. Spectrosc. Radiat. Transfer, 63: 521-543, 1999.

Chepfer, H., P. Goloub, J. Riedi, J. D. Haan, J. W. Hovenier and P. Flamant, Ice crystal shapes in cirrus clouds derived from polder/adeos-1. J. of Geophys. Res., 106: 79557966, 2000.

Chepfer, H., P. Minnis, D. Young, L. Nguyen and R. F. Arduini, Estimation of cirrus 
clouds effective ice crystals shapes using visible reflectances from dual-satellite measurements. J. of Geophys. Res., 107(D23): 21-1, 21-16, 2002.

Deschamps, P. Y., F. M. Breon, M. Leroy, A. Podaire, A. Brickaud, J. C. Buriez and G. SÃ "ze, The polder mission : instrument characteristics and scientific objectives. In IEEE Trans. Geosci. Remote Sens., 32, pp. 598-615, 1994.

Goloub, P., J. L. DeuzÃ $($, M. Herman and Y. Fouquart, Analysis of the polder polarization measurements performed over cloud covers. IEEE Transactions on geoscience and remote sensing, 32(1): 78-88, 1994.

Goloub, P., N. Herman, H. Chepfer, P. Couvert, G. Brogniez, G. Seze and J. Riedi, Cloud phase detection from polder/adeos. J. of Geophys. Res., 105: 14747-14759, 2001.

Hagolle, O., P. Goloub, P.-Y. Deschamps, H. Cosnefroy, X. Briottet, T. Bailleul, J.-M. Nicolas, F. Parol, B. Lafrance and M. Herman, Results of polder in-flight calibration. IEEE Transactions on Geoscience and Remote Sensing, 37: 1550-1566, 1999.

Hendry, A. and G. C. McCormick, Radar observations of the alignment of precipitation particles by electrostatic fields in thunderstorms. J. of Geophys. Res., 81: 5353-5357, 1976. 
Heymsfield, A. J. and C. M. R. Platt, A parametrization of the particle size spectrum of ice clouds in terms of the ambient temperature and the ice water content. J. of Atmos. Sci., 41: 846-855, 1984.

Jayaweera, K. and B. J. Mason, The behavior of freely falling cylinders and cones in a viscous fluid. J. Fluid Mech., 22: 709, 1965.

Klett, J. D., Orientation model for particles in turbulence. J. Atm. Sci., 52(12): 2276-2285, 1995.

Liou, K. N. and Y. Takano, Interpretation of cirrus cloud polarization measurements from radiative transfer theory. Geophys. Res. Let., 29(9): 27-1, 27-4, 2002.

List, R. and R. S. Schemenauer, Free-fall behavior of planar snow crystals, conical graupel and small hail. J. of Atmos. Sci., 28: 110-115, 1971.

Lynch, D. K., S. D. Gedzelman and A. B. Fraser, Subsuns, bottlinger's rings and elliptical halos. Applied Optics, 33: 4580-4589, 1994.

Magono, C., On the growth of snow flakes and graupel. Sci. Rep. Yokohama Natl Univ., Ser. 1, 2: 18-40, 1953.

Masuda, K., H. Ishimoto and T. Takashima, Retrieval of cirrus optical thickness and ice- 
shape information using total and polarized reflectance from satellite measurements.

J. of Quant. Spectrosc. Radiat. Transfer, 75: 39-51, 2002.

Noel, V., H. Chepfer, G. Ledanois and P. H. Flamant, Computation of a single-scattering matrix for nonspherical particles randomly or horizontally oriented in space. Applied Optics, 40: 4365-4375, 2001.

Noel, V., G. Roy, L. Bissonnette, H. Chepfer and P. H. Flamant, Analysis of ice clouds lidar measurements at multiple incidence angles. Geophys. Res. Let., 29: 52-1 -52-4, 2002.

Ono, A., The shape and riming properties of ice crystals in natural clouds. J. of Atmos. Sci., 26: 138-147, 1969.

Platt, C. M. R., N. L. Abshire and G. T. McNice, Some microphysical properties of an ice cloud from lidar observations of horizontally oriented crystals. J. of Appl. Met., 17: 1220-1224, 1978.

Podzimek, J., Aerodynamic conditions of ice crystal aggregation. In Proc. of Int. Conf. Cloud Physics, pp. 295-299, Amer. Meteor. Soc., Toronto, 1968. 
Riedi, J., M. Doutriaux-Boucher, P. Goloub and P. Couvert, Global distribution of cloud top phase from polder/adeos-i. Geophys. Res. Let., 27: 1707-1710, 2000.

Riedi, J., P. Goloub and R. T. Marchand, Comparison of polder cloud phase retrievals to active remote sensors measurements at the arm sgp site. Geophys. Res. Let., 28(11): 2185-2188, 2001.

Sassen, K., Remote sensing of planar ice crystals fall attitude. J. Met. Soc. of Japan, 58: 422-433, 1980.

Sassen, K. and S. Benson, A midlatitude cirrus cloud climatology from the facility for atmospheric remote sensing. part ii : microphysical properties derived from lidar depolarisation. J. of Atmos. Sci., 58: 2103-2111, 2001.

Sassen, K., K. N. Liou, Y. Takano and V. I. Khvorostyanov, Diurnal effects in the composition of cirrus clouds. Geophys. Res. Let., 30(10): 46-1,46-4, 2003.

Sassen, K. and Y. Takano, Parry arc : a polarization lidar, ray-tracing, and aircraft case study. Applied Optics, 39(36): 6738-6475, 2000.

Stephens, G. L., S.-C. Tsay, P. W. Stackhouse and P. J. Flatau, The relevance of the micro- 
physical and radiative properties of cirrus clouds to climate and climate feedback. J. of Atmos. Sci., 47: 1742-1753, 1990.

Takano, Y. and K. N. Liou, Solar radiative transfer in cirrus clouds. part 1 : Singlescattering and optical properties of hexagonal ice crystals. J. of Atmos. Sci., 46: 3-18, 1989a.

Takano, Y. and K. N. Liou, Solar radiative transfer in cirrus clouds. part 2 : Theory and computation of multiple scattering in an anisotropic medium. J. of Atmos. Sci., 46: 20-36, 1989b.

Thomas, L., J. C. Cartwright and D. P. Wareing, Lidar observations of the horizontal orientation of ice crystals in cirrus clouds. Tellus, 42B: 211-216, 1990.

Trankle, E. and R. G. Greenler, Multiple-scattering effects in halo phenomena. J. Opt. Soc. Am., 4(3): 591-599, 1987.

Van de Hulst, H. C., Light scattering by Small Particles. Dover Publications Inc., New York, 1957.

Winker, D. and B. Wielicki, The picasso-cena mission. In H. Fujisada and J. B. Lurie, editors, Sensors, Systems, and Next Generation Satellites, SPIE, pp. 26-36, 1999. 
Wylie, D. P., W. P. Menzel, H. M. Woolf and K. L. Strabala, Four years of global cirrus clouds statistics using hirs. J. of Climate, 7: 315-335, 1994. 


\section{Figure Captions}

Figure 1 : Normalized polarized radiance as a function of zenithal angle $\theta_{v}$.

Figure 2 : Local maximum as a function of maximum deviation angle $\alpha_{\max }$.

Figure 3 : Maximum normalized polarized radiance intensity as a function of crystal aspect ratio $A$.

Figure 4 : Normalized polarized radiance local maximum as a function of crystal aspect ratio $A$ and horizontally-oriented crystals relative concentration $n^{h}$.

Figure 5: Normalized polarized radiance as a function of the angle $\tau$ between the specular and scattering directions.

Figure 6 : Frequency of maximum deviation angles $\alpha_{\max }$

Figure 7 : Distribution of horizontally-oriented crystals concentrations $n^{h}$ for (a) square distribution of crystal deviation and (b) gaussian distribution of crystal deviation. 


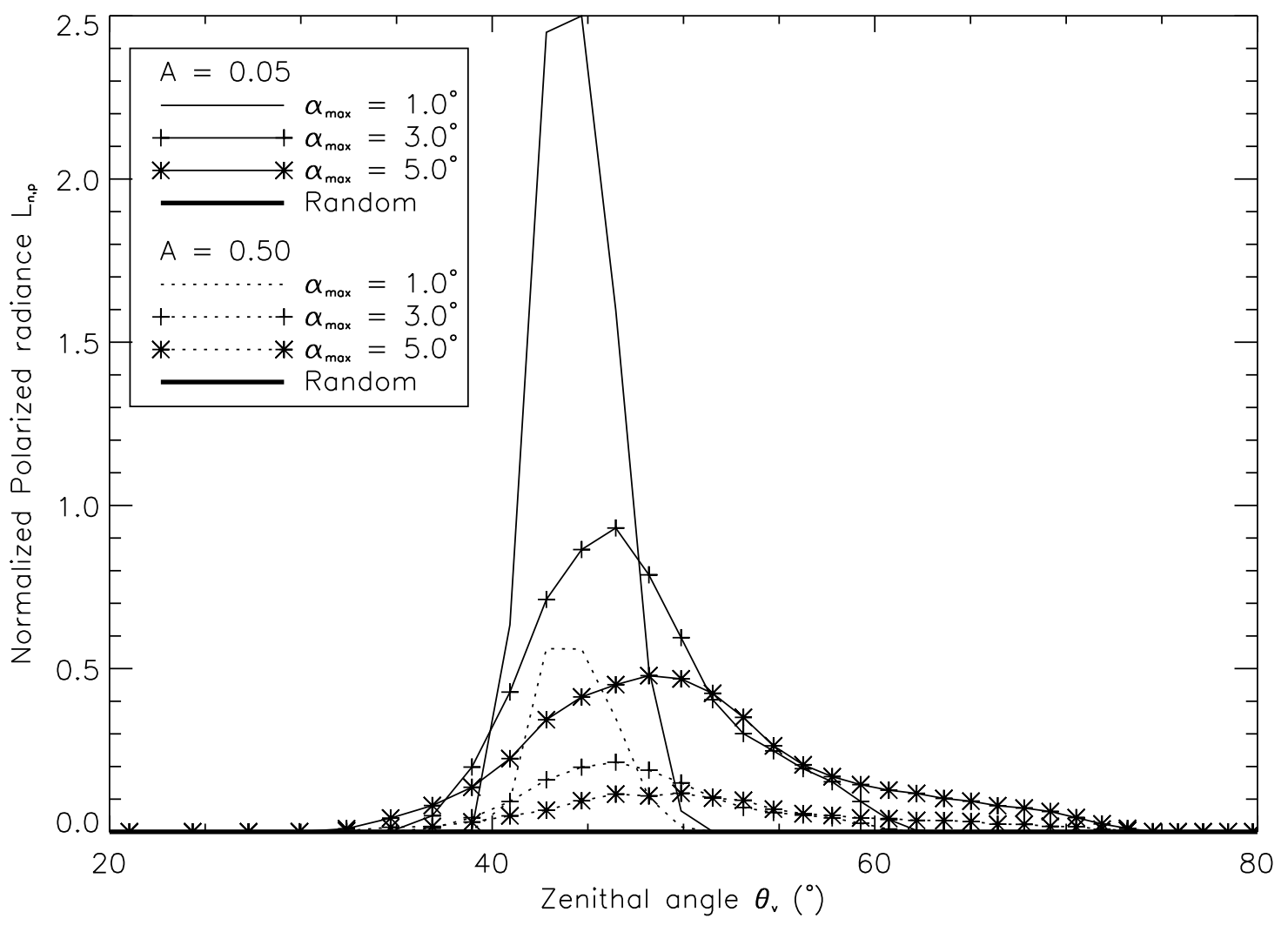

Figure 1: 


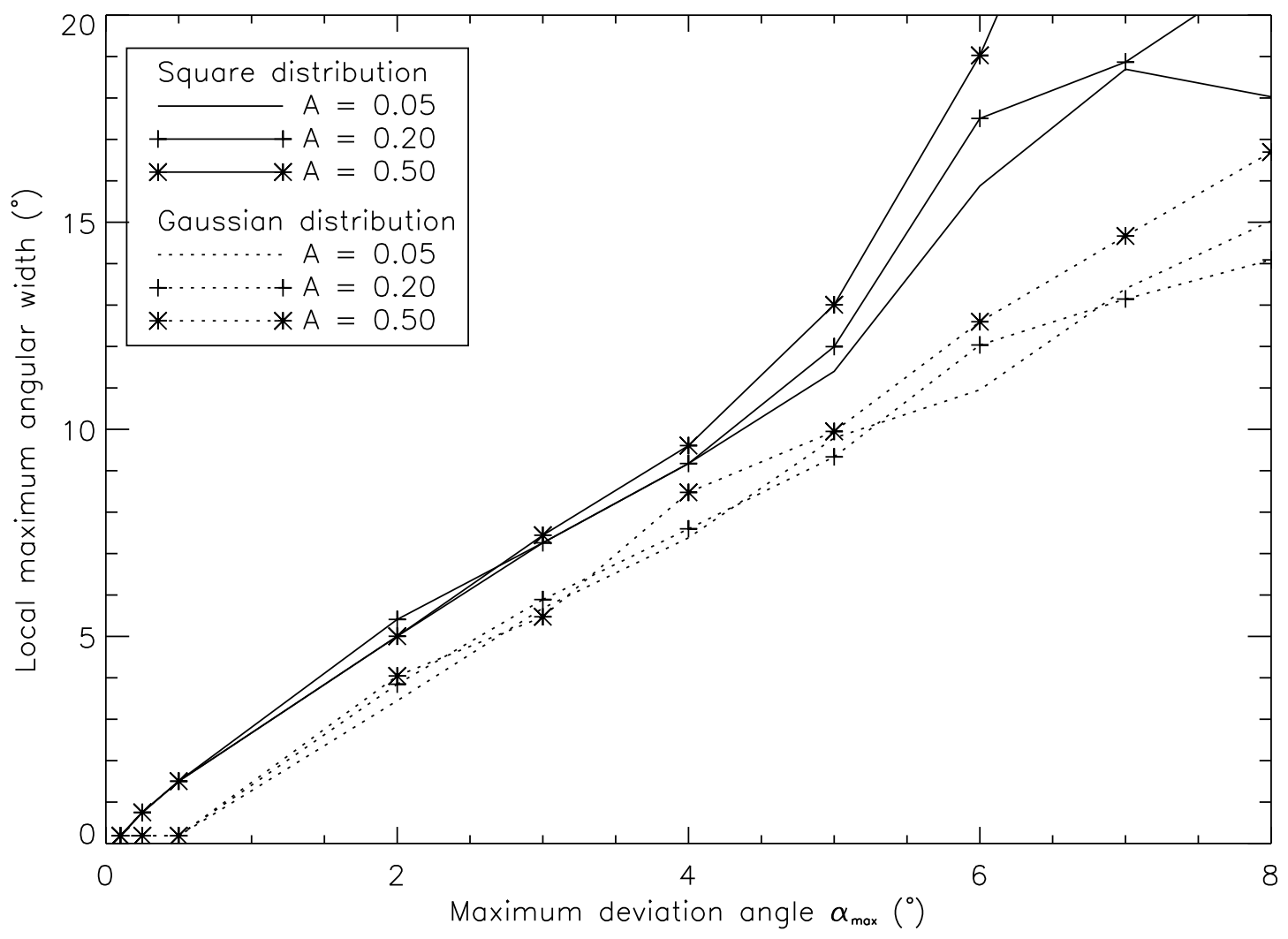

Figure 2: 


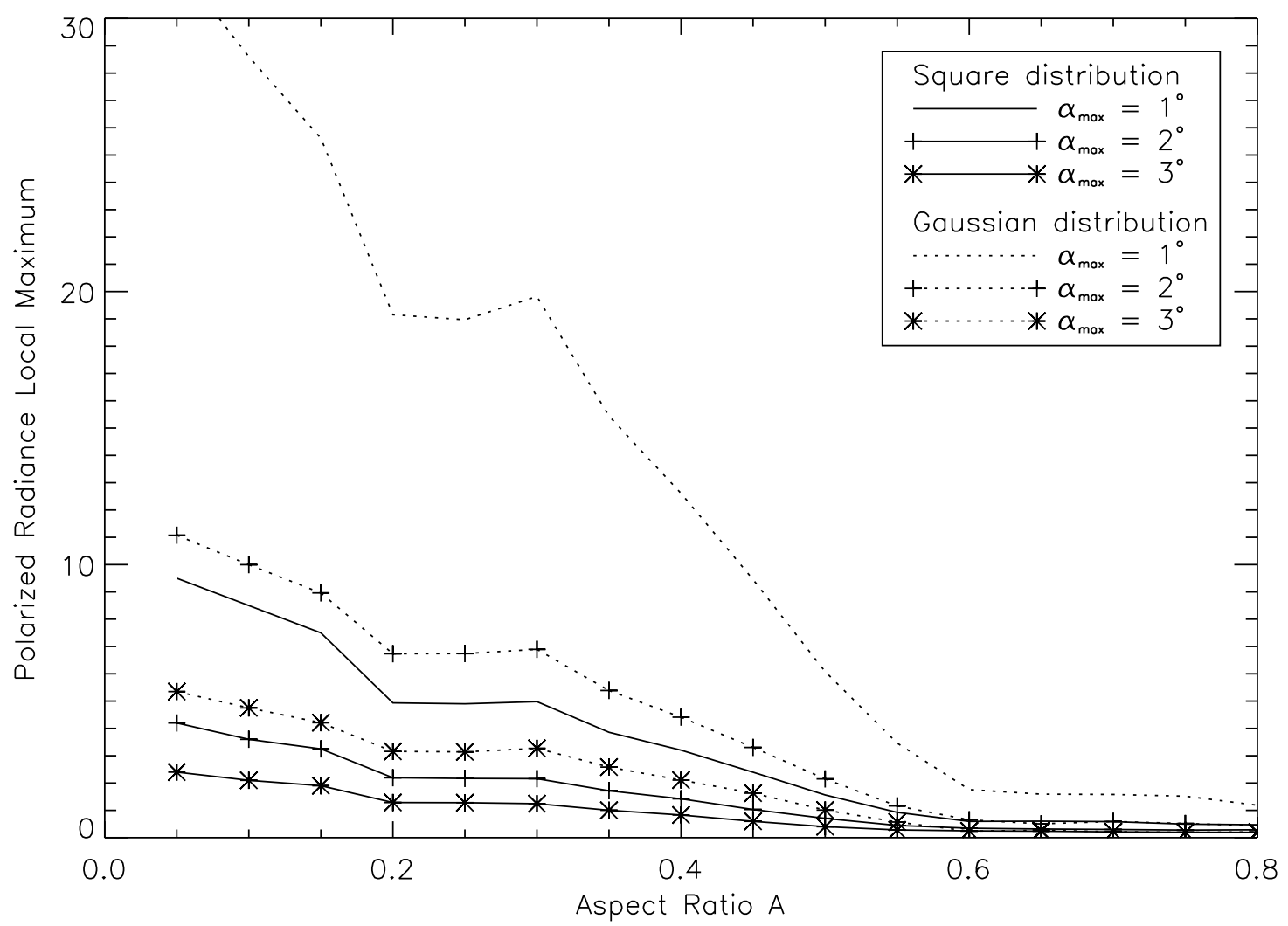

Figure 3: 


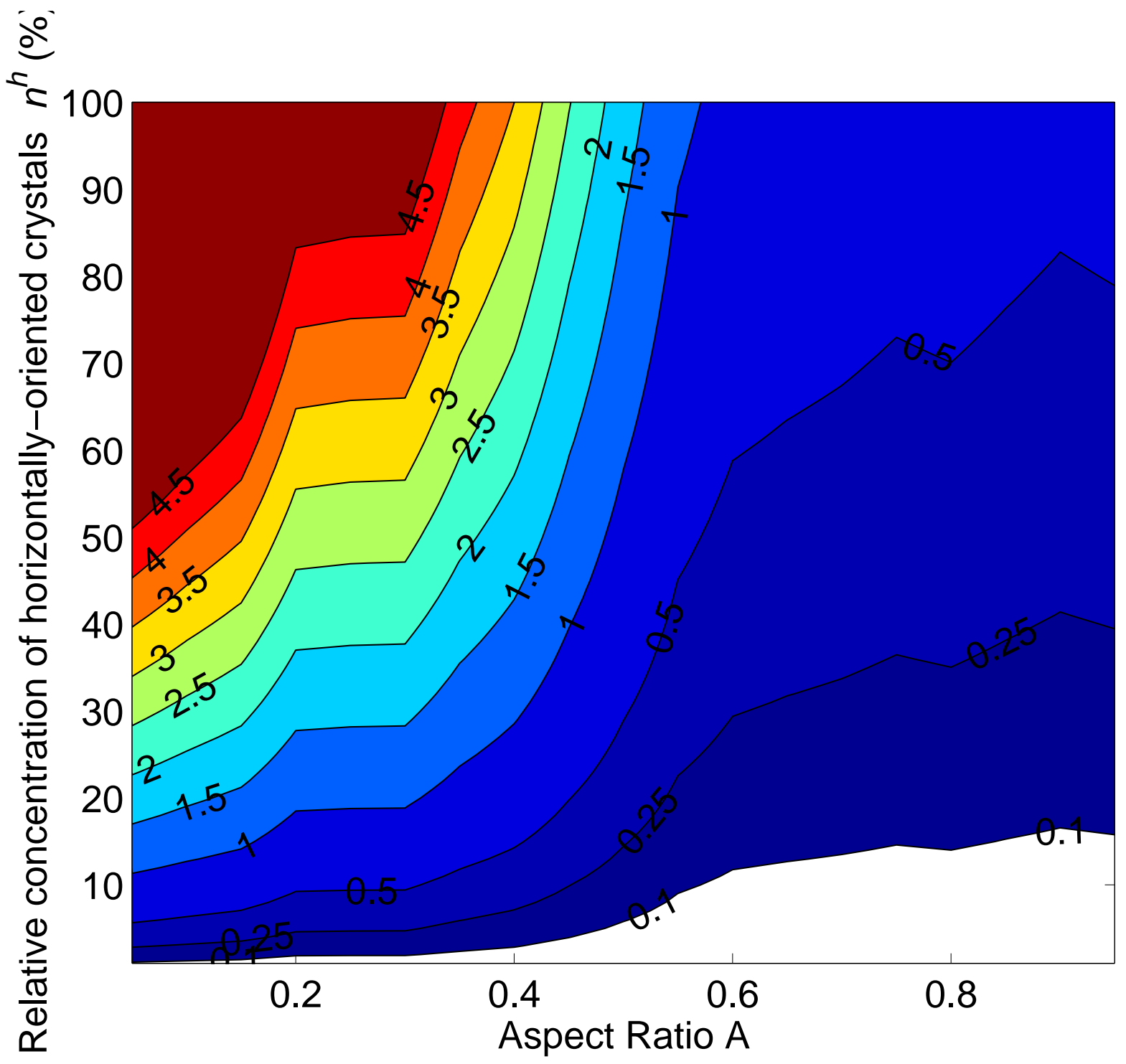

Figure 4: 


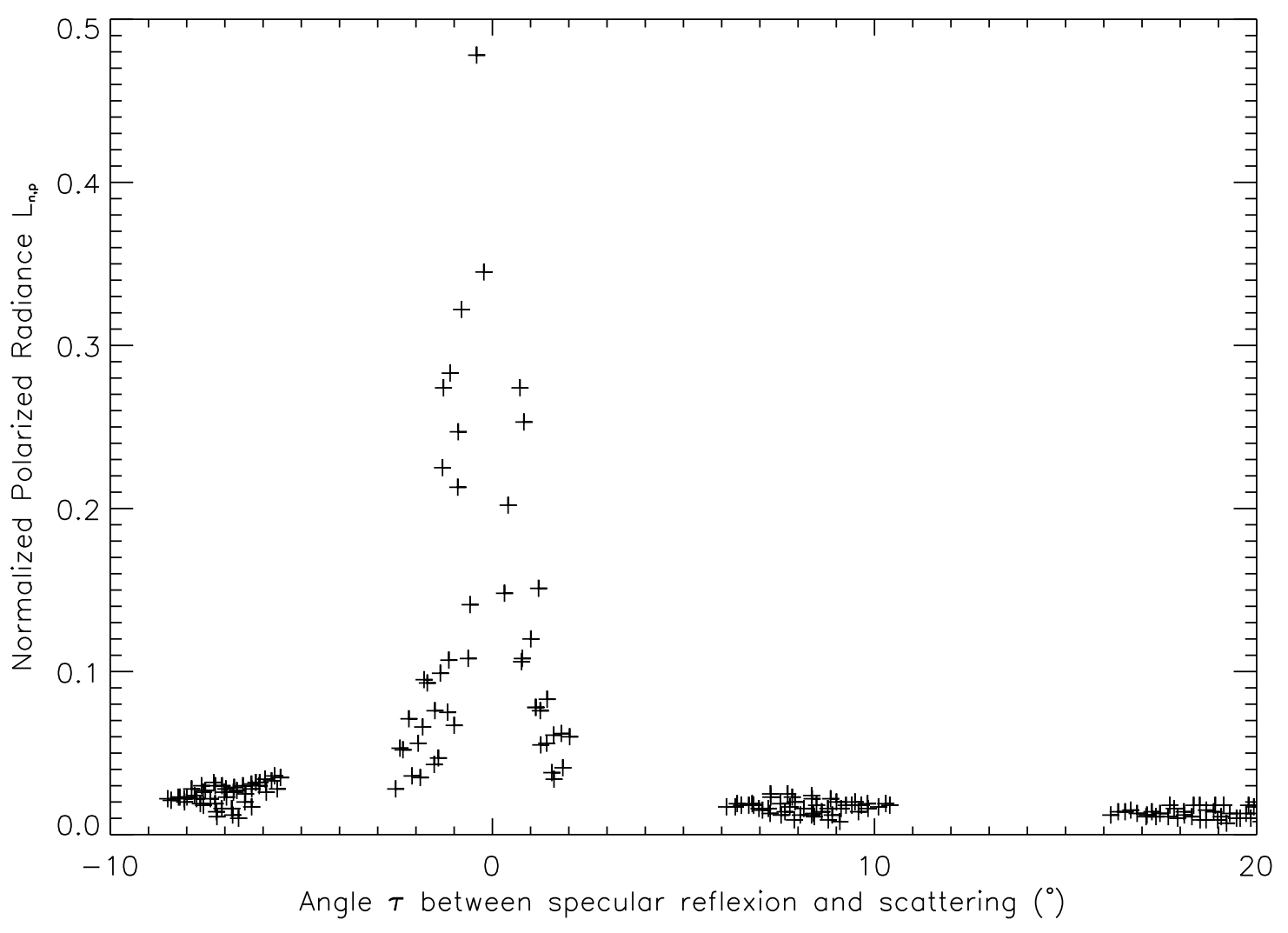

Figure 5: 


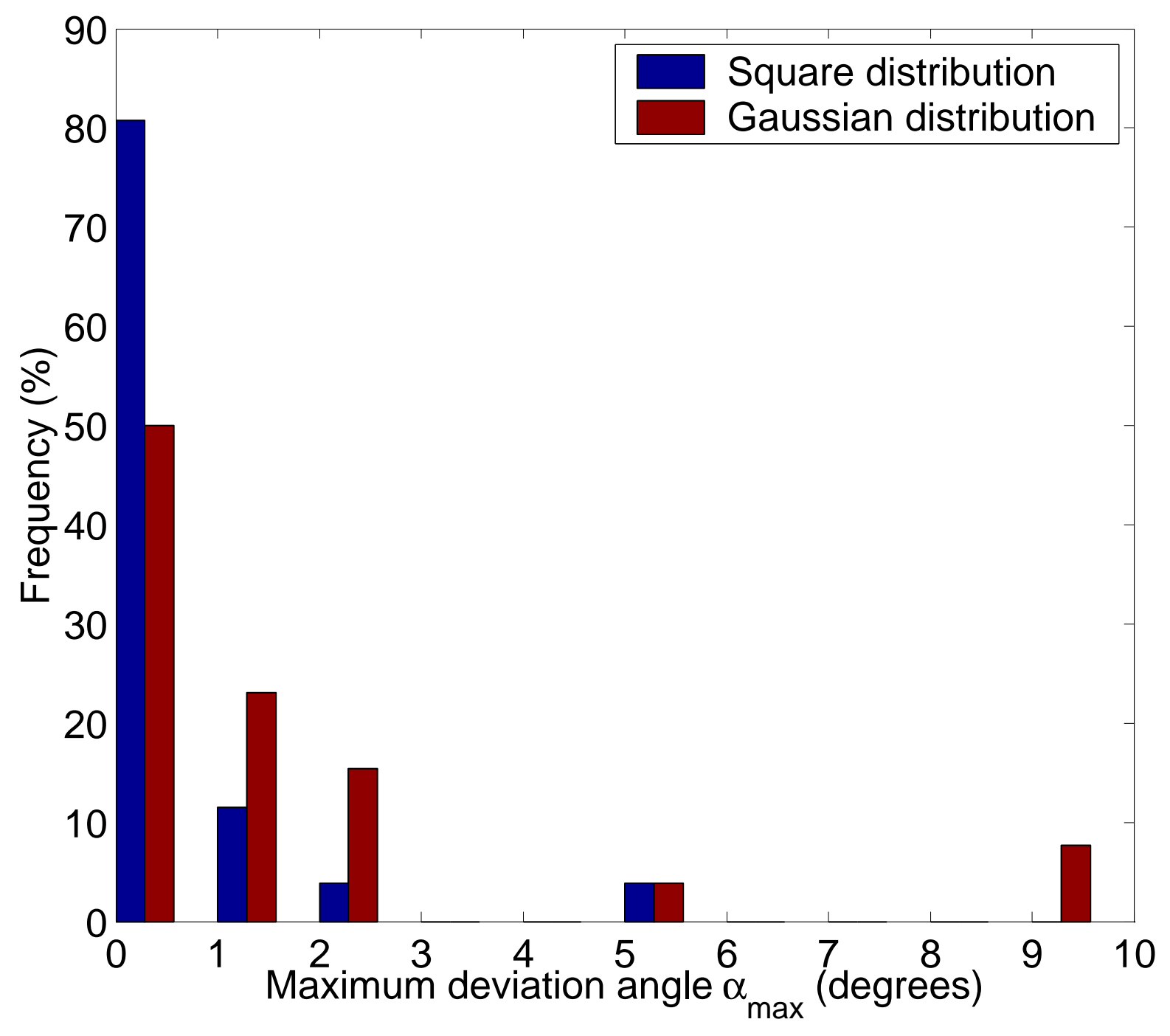

Figure 6: 

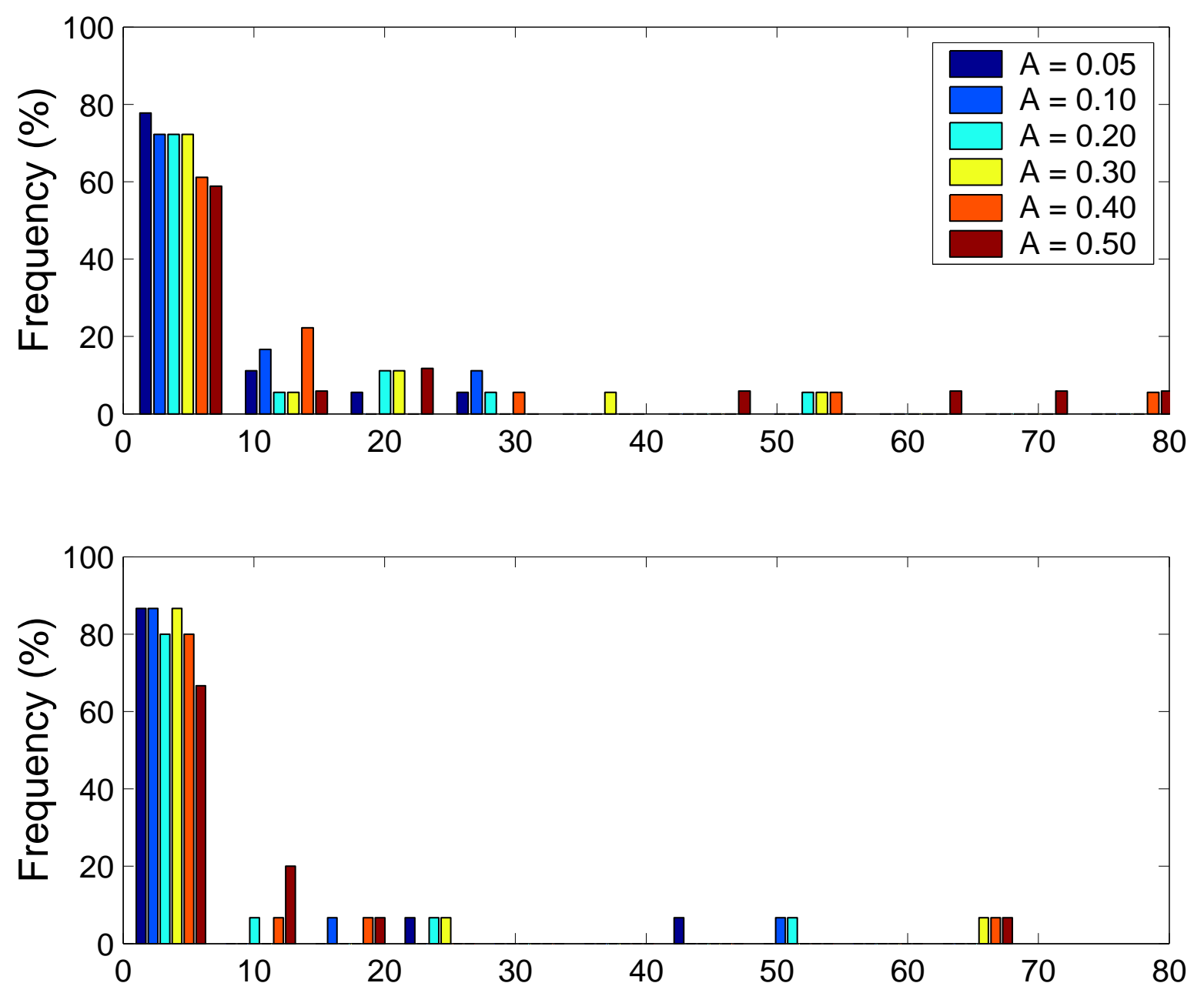

Relative concentration of horizontally-oriented particles $n^{h}(\%)$

Figure 7: 
Table Captions

Table 1 : Relative concentrations of horizontally-oriented crystals for different aspect ratios

Table 2 : Average concentrations of horizontally-oriented crystals for different aspect ratios 


\begin{tabular}{l||c|c|c|c|c|c} 
aspect ratio $A$ & 0.05 & 0.1 & 0.2 & 0.3 & 0.4 & 0.5 \\
\hline Relative concentration $n^{h}$ (square) \% & 0.7 & 0.8 & 1.1 & 1.5 & 1.7 & 3.6 \\
Relative concentration $n^{h}$ (gaussian) \% & 0.5 & 0.6 & 0.7 & 1.0 & 1.3 & 3.1
\end{tabular}

Table 1: 


\begin{tabular}{l||c|c|c|c|c|c} 
aspect ratio $A$ & 0.05 & 0.1 & 0.2 & 0.3 & 0.4 & 0.5 \\
\hline Average relative concentration $n^{h}$ (square) \% & 6.2 & 7.1 & 9.6 & 9.9 & 13.6 & 21.4 \\
Average relative concentration $n^{h}$ (gaussian) \% & 5.4 & 6.0 & 6.7 & 7.2 & 8.0 & 8.6
\end{tabular}

Table 2: 